\title{
Dihydroartemisinin inhibits multiplication of Brucella suis vaccine strain 2 in murine microglia BV2 cells via stimulation of caspase-dependent apoptosis
}

\author{
JUAN YANG $^{1 *}$, HAINING LI ${ }^{1 *}$, ZHAO WANG ${ }^{1 *}$, LIMING YU ${ }^{1}$, QIANG LIU ${ }^{1}$, \\ XIAOYAN NIU ${ }^{1}$, TING XU ${ }^{1}$ and ZHENHAI WANG ${ }^{1,2}$ \\ ${ }^{1}$ Department of Neurology, The General Hospital of Ningxia Medical University; \\ ${ }^{2}$ Ningxia Key Laboratory of Cerebrocranial Diseases, Incubation Base of National Key Laboratory, \\ Yinchuan, Ningxia Hui Autonomous Region 750004, P.R. China
}

Received November 28, 2018; Accepted July 26, 2019

DOI: $10.3892 / \mathrm{mmr} .2019 .10672$

\begin{abstract}
Brucellosis, caused by a facultative intracellular parasite Brucella species, is the most common bacterial zoonotic infection worldwide. Brucella can survive and proliferate in several phagocytic and non-phagocytic cell types. Human brucellosis has similar clinical symptoms with systemic diseases, which may lead to delay of diagnosis and increasing of complications. Therefore, investigating the proliferation of Brucella in host cells is important to understand the pathogenesis of the disease. Dihydroartemisinin (DHA), a semi-synthetic derivative of artemisinin, has been recommended by World Health Organization as an anti-malarial drug. However, there have been few studies regarding its effectiveness against bacteria. In the present study, it was revealed that $B$. suis vaccine strain 2 (S2) grew in BV2 cells without significant cytotoxicity, and less than $20 \mu \mathrm{M}$ DHA had no inhibitory effects on BV2 cells. Furthermore, DHA reduced B. suis S2 growth in BV2 cells, and increased the percentage of apoptosis and the expression of cleaved caspase-3 in B. suis S2-infected cells. Collectively, the present data indicated that DHA induced the caspase-dependent apoptotic pathway to inhibit the intracellular $B$. suis $\mathrm{S} 2$ growth.
\end{abstract}

\section{Introduction}

Brucellosis, caused by a facultative intracellular parasite Brucella species, is the most common bacterial zoonotic

Correspondence to: Dr Zhenhai Wang, Department of Neurology, The General Hospital of Ningxia Medical University, 804 South Shengli Street, Yinchuan, Ningxia Hui Autonomous Region 750004, P.R. China

E-mail: wangzhenhai1968@163.com

*Contributed equally

Key words: dihydroartemisinin, B. suis s2, BV2 cells, apoptosis, caspase-3 infection worldwide $(1,2)$. Brucella can survive and proliferate in several phagocytic and non-phagocytic cell types, including macrophages, dendritic cells, placental trophoblasts in vivo, and has the ability to replicate in a variety of mammalian cells, such as microglia, fibroblasts, epithelial and endothelial cells (3). In China, Brucellosis represents a major challenge from the last decade, and a high incidence of human brucellosis has been reported in northwestern China (4). Human brucellosis has similar clinical symptoms with systemic diseases and can lead to delay in diagnosis and may increase unexpected complications (5). Therefore, investigating the proliferation of Brucella in host cells is important to understand the pathogenesis of the disease. In addition, earlier evidence indicates that Brucella inhibits apoptosis of host cells to maintain an intracellular niche for its replication (6). Therefore, induction of apoptosis in the host cells may provide a useful therapeutic strategy for the treatment of Brucellosis.

Dihydroartemisinin (DHA), a semi-synthetic derivative of artemisin, has been recommended by World Health Organization as an anti-malarial drug (7). Previous studies have documented that DHA possesses the ability to prevent angiogenesis (8), cause cell cycle arrest (9), promote apoptosis, and inhibit cancer invasion (10). However, there have been few studies regarding its effectiveness against bacteria.

In the present study, it was investigated whether DHA inhibited the intracellular growth of Brucella suis vaccine strain 2 (B. suis S2) in murine microglia BV2 cells, and whether the inhibitory effect of DHA was associated with stimulation of apoptosis of BV2 cells.

\section{Materials and methods}

Cell line and bacteria. Murine microglia BV2 cells were obtained from China Center for Type Culture Collection and cultured with Dulbecco's modified Eagle's medium (DMEM; Gibco; Thermo Fisher Scientific, Inc.) containing $10 \%$ fetal bovine serum (FBS; Hyclone; GE Healthcare Life Sciences), $2 \mathrm{mM}$ glutamine, and $200 \mathrm{mM}$ streptomycin/penicillin and maintained in $5 \% \mathrm{CO}_{2}$ at $37^{\circ} \mathrm{C}$. B. suis $\mathrm{S} 2$ was provided by Professor $\mathrm{Xu}$ from Ningxia 

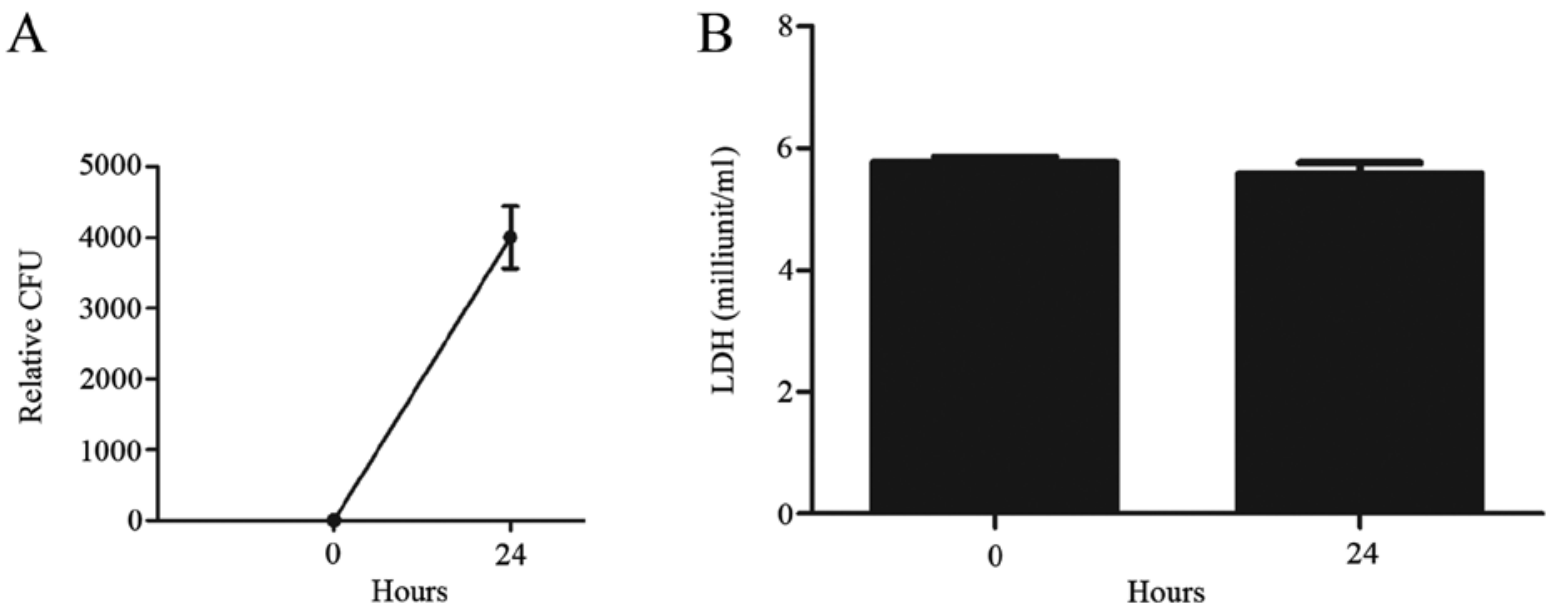

Figure 1. Growth of B. suis S2 in BV2 cells and cellular LDH release. (A) Cells were infected with B. suis S2 by MOI 100 for $24 \mathrm{~h}$. Growth of bacteria was evaluated by CFU assay. Data are reported as the mean \pm SD. (B) Supernatants of BV2 cells from indicated groups were collected and evaluated for LDH. Data are reported as the mean $\pm \mathrm{SD}$. B. suis $\mathrm{S} 2, B$. suis vaccine strain 2; $\mathrm{LDH}$, lactate dehydrogenase; MOI; multiplicity of infection; $\mathrm{CFU}$, colony-forming unit.
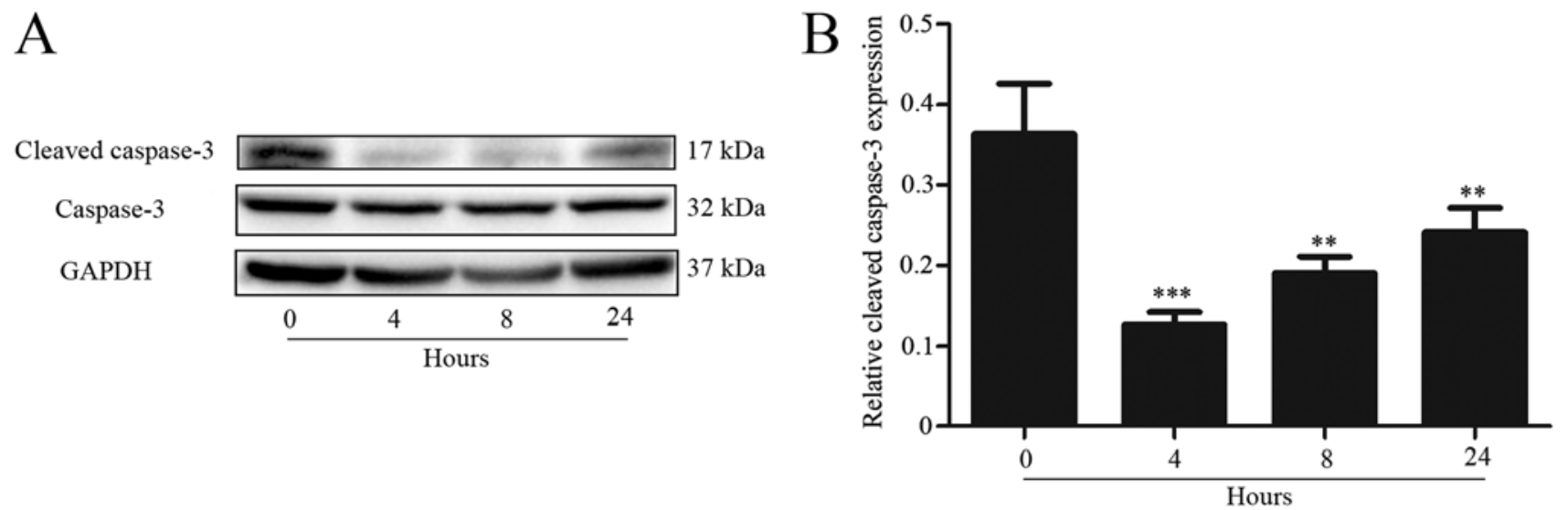

Figure 2. Western blot analysis of caspase-dependent apoptosis-associated proteins. (A) Representative blots of three independent experiments are presented, and the protein size is expressed in $\mathrm{kDa}$. (B) Quantification of cleaved caspase-3 protein levels from three separate experiments, normalized to GAPDH. ${ }^{* *} \mathrm{P}<0.01,{ }^{* * *} \mathrm{P}<0.001$ vs. BV2 control cells.

Medical University (Yinchuan, China) and was grown in Soybean-Casein Digest Agar Medium (TSA) at $37^{\circ} \mathrm{C}$ in a 5\% $\mathrm{CO}_{2}$ incubator. The single $B$. suis $\mathrm{S} 2$ colony was inoculated in sterilized tryptic soy broth (TSB) solution at $37^{\circ} \mathrm{C}$ in a $5 \% \mathrm{CO}_{2}$ atmosphere until its use. Bacteria were harvested by centrifugation for $20 \mathrm{~min}$ at $2,000 \mathrm{x}$ g at $4^{\circ} \mathrm{C}$ and washed twice with phosphate-buffered saline (PBS). Bacterial density in the cultures were estimated by the McFarland standards of bioMérieux, Inc. All bacterial experiments were carried out at the Biosafety Level 2 Laboratory (Key Laboratory of Pathogenic Microorganisms, The general hospital of Ningxia Medical University).

In vitro infection and DHA treatment. A total of $2 \times 10^{5} \mathrm{BV} 2$ cells were seeded in 6-well culture plates. After reaching $60 \%$ confluence, the cells were exposed to B. suis S2 at a multiplicity of infection (MOI) of 100 for $2 \mathrm{~h}$ in medium without antibiotics. Subsequently, BV2 cells were extensively washed to remove extracellular bacteria, and infection was maintained for $24 \mathrm{~h}$ in the presence of $100 \mu \mathrm{g} / \mathrm{ml}$ gentamicin to kill remaining extracellular bacteria (11). Two hours after infection, the cells were treated with different concentrations of DHA $(0,10,20,30$ and $40 \mu \mathrm{M})$.

Colony-forming unit assay (CFU assay). The TSA was prepared according to the manufacturer's instructions and autoclaved at $120^{\circ} \mathrm{C}$ for $30 \mathrm{~min}$. On a clean bench, $\sim 25 \mathrm{ml}$ of TSA was poured into a $90-\mathrm{mm}^{2}$ sterile culture plate, inverted after coagulation, and used for bacterial culture. BV2 cells were infected with B. suis S2 at MOI 100 for $24 \mathrm{~h}$, washed with PBS twice and lysed in $0.3 \%$ Triton-X100 for $10 \mathrm{~min}$. The sample was inoculated on TSA media for $72 \mathrm{~h}$, and the cell suspension was diluted to count the number of colonies. The visible colonies with a diameter greater than $1 \mathrm{~mm}$ were manually counted with an optical microscope.

Cell viability assay. Cell viability was determined by Cell-counting Kit-8 (CCK-8; BestBio) assay according to the manufacturer's protocol (12). In brief, cells $\left(1 \times 10^{4}\right)$ were seeded in 96-well culture plates and cultured overnight. The cells were treated with different concentrations of DHA $(0,10$, 20,40 and $80 \mu \mathrm{M}$ ) for $24 \mathrm{~h}$, and the CCK- 8 solution was added 

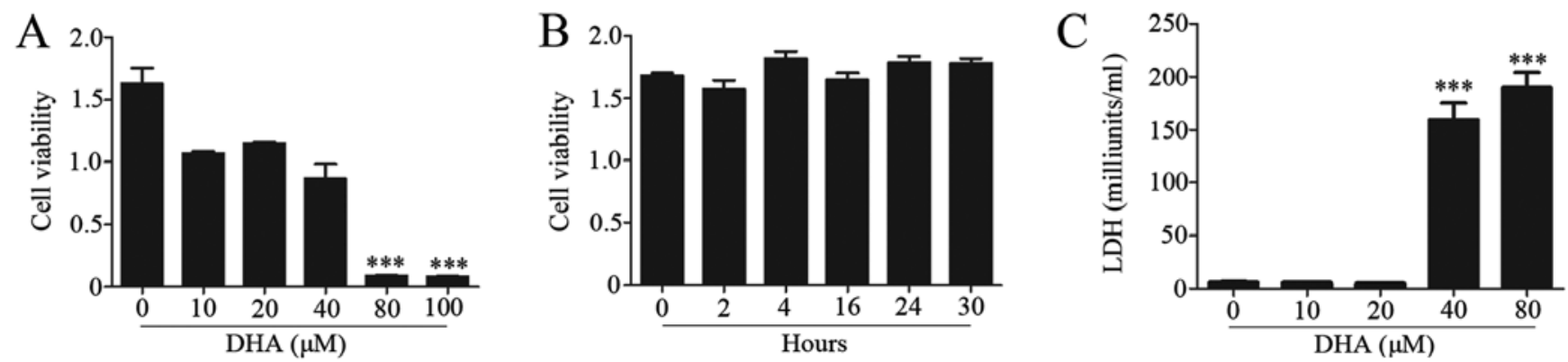

Figure 3. Effects of DHA on BV2 cells. (A) Cells were treated with various concentrations of DHA for 24 h, and cell viability was evaluated by CCK-8 assay. (B) Cells were treated with $20 \mu \mathrm{M}$ DHA for indicated time-points, and cell viability was evaluated by CCK- 8 assay. (C) Cells were treated with various concentrations of DHA for $24 \mathrm{~h}$, and cell toxicity was evaluated by LDH release. ${ }^{* * *} \mathrm{P}<0.001$ vs. the control. DHA, dihydroartemisinin; LDH, lactate dehydrogenase.

to each well and incubated for an additional $4 \mathrm{~h}$. The absorbance at $450 \mathrm{~nm}$ was measured by Microplate reader (BioTek Instruments, Inc.) (13).

Lactate dehydrogenase $(\mathrm{LDH})$ assay. The toxicity of BV2 cells after $B$. suis $\mathrm{S} 2$ infection was determined by the LDH assay using a commercially available kit (Sigma-Aldrich; Merck KGaA) (14). Briefly, the BV2 cells were infected with B. suis S2 at MOI 100 or exposure to different concentrations of DHA $(0,10,20,30$ and $40 \mu \mathrm{M})$ for $24 \mathrm{~h}$. Finally, the supernatant was collected for the LDH assay.

Flow cytometric assay. The BV2 cells were infected with $B$. suis $\mathrm{S} 2$ and then stained with Annexin V/PI according to manufacturer's protocol (BestBio). The apoptosis in cells was analyzed by flow cytometric analysis (FCM).

Western blot analysis. Cells were harvested, washed twice with PBS, and lysed in RIPA buffer (Nanjing KeyGen Biotech Co., Ltd.). Protein concentration in the lysates was determined by the bicinchoninic acid (BCA) method according to the manufacturer's recommendations (Nanjing KeyGen Biotech Co., Ltd.). Protein $(30 \mu \mathrm{g})$ was separated on $12 \%$ Mini-Protean TGX gels and subsequently transferred onto a polyvinylidene difluoride (PVDF) membrane according to standard protocols. Caspase-3 (ID product code ab184787; dilution in 1:1,000), cleaved caspase-3 (ID product code ab49822; dilution in 1:500) or GAPDH (ID product code ab181602; dilution in 1:5,000; All from Abcam) antibodies were used for protein detection. Goat anti-mouse polyclonal antibody (Beijing Zhongshan Golden Bridge Biotechnology Co., Ltd.; OriGene Technologies; ZB-2305; dilution 1:5,000) was used as a secondary antibody. The blot was developed using the Western Lightning Ultra chemiluminescent substrate from Bio-Rad Laboratories, Inc., and detected using EpiChemi3 darkroom (UVP BioImaging Systems).

Antimicrobial susceptibility testing. The single clone was added to $5 \mathrm{ml} \mathrm{TSB}$, incubated in a $37^{\circ} \mathrm{C}$ incubator for $24 \mathrm{~h}$, and the concentration of the bacteria suspensions was adjusted to $\sim 10^{8} \mathrm{CFU} / \mathrm{ml}$ with TSB. TSB $(200 \mu \mathrm{l})$ and bacteria suspensions were used as a negative control and positive control, respectively. After loading the drug and bacteriostatic solution, the plate was incubated at $37^{\circ} \mathrm{C}$ in an incubator for $24 \mathrm{~h}$, and the absorbance at $580 \mathrm{~nm}$ was measured (15).

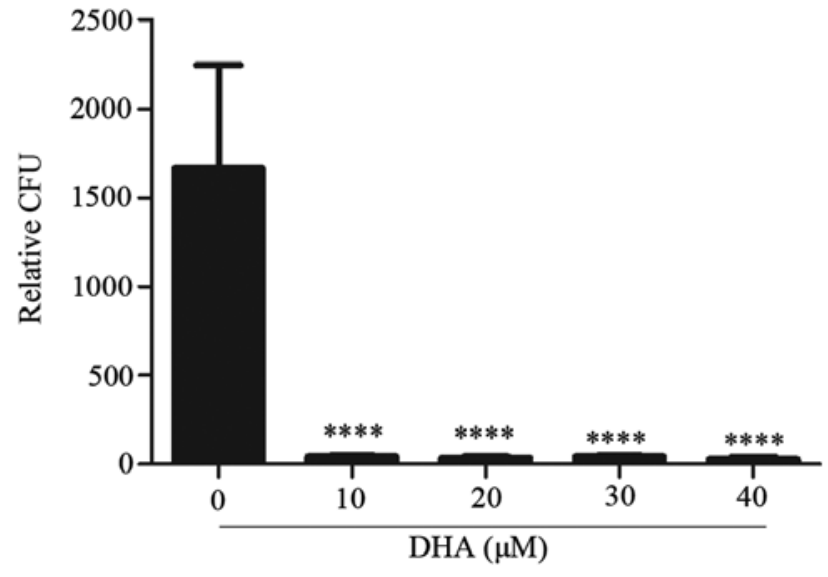

Figure 4. Effects of DHA on the B. suis $\mathrm{S} 2$ growth in BV2 cells. DHA significantly inhibited the growth of $B$. suis $\mathrm{S} 2$ in BV2 cells. ${ }^{* * * *} \mathrm{P}<0.0001$ vs. the untreated BV2-infected group. DHA, dihydroartemisinin; B. suis $\mathrm{S} 2$, B. suis vaccine strain 2 .

Statistical analysis. All data are presented as the mean \pm standard deviation (SD) from at least three independent experiments. GraphPad Prism 7.0 (GraphPad Software) was used for statistical analysis. Statistical differences between indicated groups were performed using one-way analysis of variance (ANOVA) followed by post hoc Tukey's test. $\mathrm{P}<0.05$ was considered to indicate a statistically significant difference.

\section{Results}

Replication of B. suis $S 2$ in murine microglia BV2 cells. In order to investigate the growth of B. suis S2 in BV2 cells, BV2 cells were infected in vitro with MOI 100. It was revealed that there was a significant intracellular increase in CFUs $24 \mathrm{~h}$ post-infection (Fig. 1A), indicating the active growth of $B$. suis $\mathrm{S} 2$ in the murine microglial cells. In addition, there was no apoptosis of BV2 cells under light microscopy (data not shown) after bacterial infection, and the levels of LDH were not significantly altered $24 \mathrm{~h}$ post-infection (Fig. 1B).

Inhibition of caspase-dependent apoptosis after B. suis S2 infection. Western blot analysis revealed that the expression of cleaved caspase-3 was significantly decreased after $B$. suis $\mathrm{S} 2$ infection, but gradually increased as the infection period 


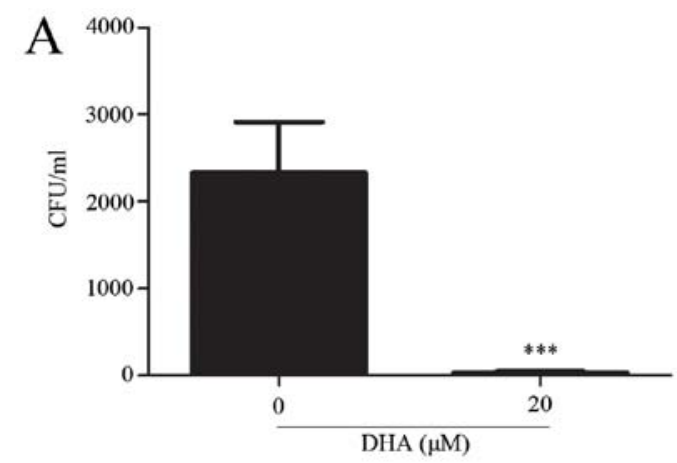

$\mathrm{B}$
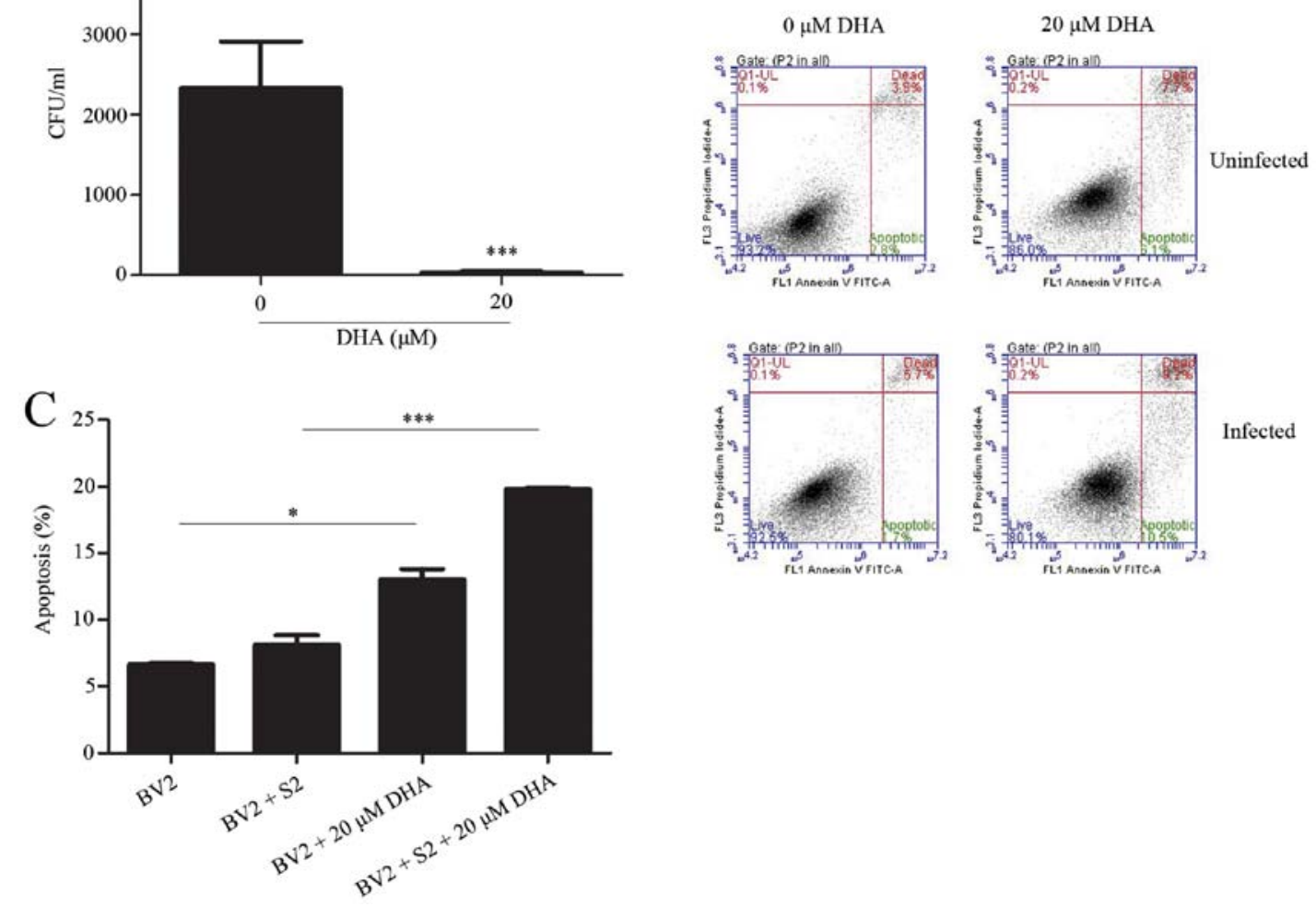

Figure 5. Effects of $20 \mu \mathrm{M}$ DHA on intracellular B. suis S2 multiplication and BV2 cellular apoptosis. (A) The effects of $20 \mu \mathrm{M}$ DHA on the B. suis S2 growth in BV2 cells. (B) Percentage of BV2 cell apoptosis was determined by flow cytometric assay. Different quadrants exhibit the percentage of viable cells as determined by scattering profiles of flow cytometric assay. (C) Values are expressed as the means \pm SD of three individual experiments. ${ }^{*} \mathrm{P}<0.05{ }^{, * * *} \mathrm{P}<0.001$. DHA, dihydroartemisinin; B. suis $\mathrm{S} 2$, B. suis vaccine strain 2; CFU, colony-forming unit.

increased (Fig. 2). The present data indicated that Brucella may inhibit apoptosis of host cells for the infection.

Effects of DHA on BV2 cell viability. To observe the biological effects of DHA on BV2 cells, the cells were treated with different concentrations of DHA for $24 \mathrm{~h}$. The results revealed that concentrations $<40 \mu \mathrm{M}$ DHA exhibited no inhibitory effects on cell viability (Fig. 3A). Moreover, cells were treated with $20 \mu \mathrm{M}$ DHA for various intervals, and no significant changes in cell viability were observed (Fig. 3B). The LDH assay demonstrated that a dose of DHA $<20 \mu \mathrm{M}$ had no inhibitory effects on BV2 cells (Fig. 3B).

$D H A$ reduces $B$. suis $S 2$ growth in $B V 2$ cells. The present data revealed that intracellular growth of $B$. suis $\mathrm{S} 2$ could inhibit cell apoptosis of BV2 cells (unpublished data). Furthermore, CFU assay results of the treated BV2-infected cells with 10-40 $\mu \mathrm{M}$ DHA revealed a significant and marked reduction in the $B$. suis S2 growth in the BV2 cells (Fig. 4).

DHA (20 $\mu M)$ inhibits bacterial multiplication by inducing apoptosis. The effects of DHA on B. suis S2 multiplication in BV2 cells were analyzed by the flow cytometric assay. The results revealed that $20 \mu \mathrm{M}$ DHA treatment increased the level of apoptosis in B. suis S2-infected BV2 cells (Fig. 5). Furthermore, western blot analysis revealed that $20 \mu \mathrm{M}$ of DHA treatment increased the expression of cleaved caspase-3 in B. suis $\mathrm{S} 2$-infected BV2 cells (Fig. 6). The present data indicated that DHA inhibited bacterial multiplication through induction of apoptosis in BV2 cells.

DHA does not directly inhibit or kill B. suis $S 2$. To confirm whether DHA affects $B$. suis S2 growth directly or indirectly through apoptosis, an antibacterial susceptibility test was performed. The results revealed that different concentrations of DHA had no effect on bacterial growth (Fig. 7), indicating that DHA does not directly inhibit or kill $B$. suis S2. DHA did not exhibit any direct effects on bacterial growth, which supports our theory that the mechanism involved in the reduction of intracellular replication of BV2 cells by DHA may be achieved by promoting apoptosis of BV2 cells.

\section{Discussion}

Brucella is an intracellular bacterium that can cause chronic infections. It has been reported that infection of Brucella inhibits apoptosis of host cells $(16,17)$. Therefore, it is necessary to investigate the mechanism underlying infection and develop therapeutic strategies for Brucellosis.

In the present study, it was demonstrated that $B$. suis $\mathrm{S} 2$ grew in BV2 cells without significant cytotoxicity, which is consistent with the characteristics of intracellular parasites. Previous studies revealed that Brucella outer membrane protein OMP25 inhibited apoptosis of host cells (6). Similarly, this study confirmed that the caspase-dependent apoptotic 
A

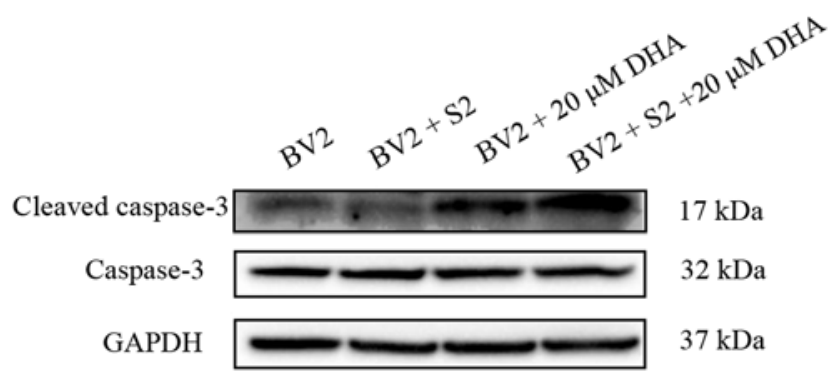

$\mathrm{B}$

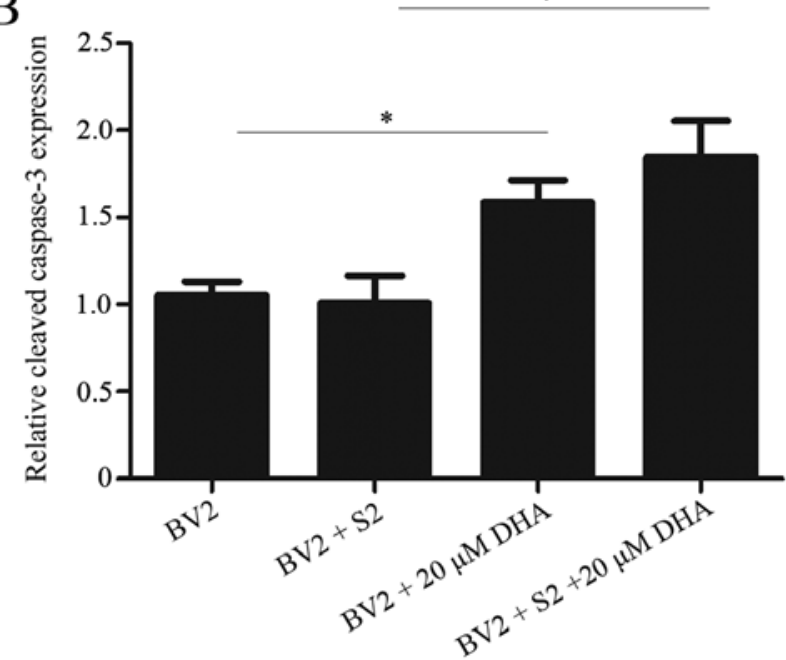

Figure 6. Western blot analysis of the caspase-dependent apoptosis in B. suis S2-infected BV2 cells. (A) Representative blots of three independent experiments are presented, and the protein size is expressed in $\mathrm{kDa}$. (B) Densitometric quantification data are expressed as the intensity ratio of target proteins to GAPDH. ${ }^{*} \mathrm{P}<0.05$. CFU, colony-forming unit; DHA, dihydroartemisinin; B. suis $\mathrm{S} 2$, B. suis vaccine strain 2.

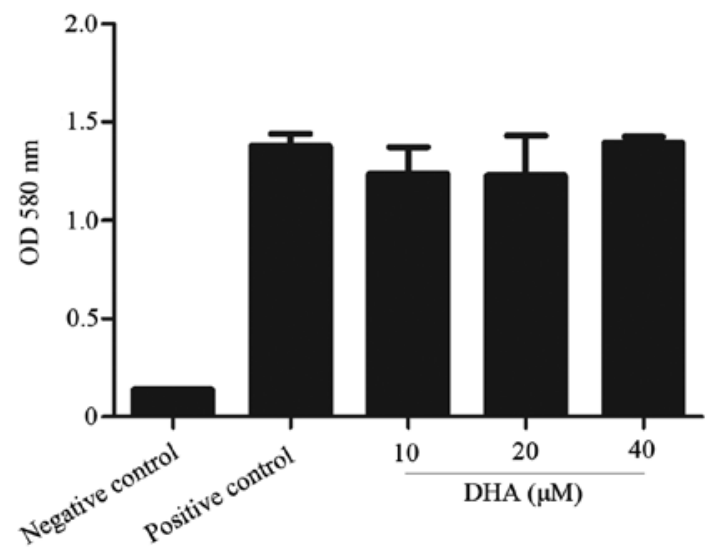

Figure 7. Antibacterial susceptibility test performed for the B. suis S2-infected cells. Cells were treated with indicated concentrations of DHA. TSB $(200 \mu \mathrm{l})$ and bacteria suspensions were used as a negative control and a positive control, respectively. B. suis $\mathrm{S} 2, B$. suis vaccine strain 2; DHA, dihydroartemisinin; TSB, tryptic soy broth.

pathway of BV2 cells was significantly inhibited after $B$. suis $\mathrm{S} 2$ infection, which provides a favorable condition for the intracellular replication of $B$. suis $\mathrm{S} 2$. This result is consistent with our findings on the mechanism of sustained infection for intracellular parasites, such as Mycobacterium tuberculosis (18).

Apoptosis, programmed cell death, is indispensable for the development and homeostasis of multicellular organisms (19). Caspase-3 is one of the activated caspases that catalyzes the cleavage of many key cellular proteins during apoptosis (20). In the present study, western blot analysis revealed that the expression of cleaved caspase- 3 was significantly decreased in BV2 cells after B. suis S2 infection, but gradually increased as the infection period increased. These observations indicated that Brucella may inhibit apoptosis of host cells for the infection. The present results are in agreement with the findings of
Brucella in other types of cells (21). Collectively, these data indicated that inhibition of host cell apoptosis may be a common strategy for Brucella-sustained infection. The underlying mechanisms of the DHA effect on bacteria remain unknown. Some studies suggest that the strategy of Brucella for infection establishment includes the following: i) to avoid intracellular destruction by limiting the fusion of the type IV secretion system-dependent vacuole-containing vacuoles to lysosomes; ii) inhibiting apoptosis of infected monocytes; and iii) inhibition of dendritic cell maturation, inhibition of antigen presentation and activation of naive $\mathrm{T}$ cells (22). It is our aim to investigate the mechanisms in our future studies.

Conversely, DHA displays potent anti-viral and anti-parasitic activities $(23,24)$, however, the molecular mechanisms are not fully understood yet. Artemisinin was also effective on bacteria. For example, oral administration of artesunate was revealed to reduce death from sepsis caused by E. coli $(25,26)$. In combination with antibiotic oxacillin, oral administration of artesunate revealed a high effectiveness against mouse sepsis induced by methicillin-resistant aureus (MRSA). On the basis of previous findings, the inhibitory effects of DHA were evaluated on B. suis $\mathrm{S} 2$ in vitro. It was revealed that DHA inhibited $B$. suis $\mathrm{S} 2$ growth in BV2 cells. Furthermore, DHA increased the percentage of apoptosis and the expression/proteolytic degradation of caspase-3 in B. suis $\mathrm{S} 2$-infected cells. It is therefore concluded that DHA induces caspase-dependent apoptotic pathway activation to inhibit intracellular B. suis S2 growth.

Brucella is an intracellular parasitic bacterium. In the present study, BV2 cells were infected with B. suis S2, and CFU experiments revealed that $B$. suis $\mathrm{S} 2$ grows in BV2 cells. After treatment with DHA, apoptosis of BV2 cell was increased. Additional experiments demonstrated that DHA had no direct effects on B. suis S2, which is similar to the effect of antibiotics on bacteria. Therefore, it is concluded that the mechanism involved in the reduction of intracellular replication of BV2 cells by DHA may be achieved by promoting apoptosis of BV2 cells. 


\section{Acknowledgements}

Not applicable.

\section{Funding}

The present study was supported by grants from the National Natural Foundation of China (grant no. 31660030) and the First-Class Discipline Construction Project in Colleges and Universities of Ningxia (grant no. NXYLXK2017A05).

\section{Availability of data and materials}

The datasets used and/or analyzed during the current study are available from the corresponding author on reasonable request.

\section{Authors' contributions}

JY, HL, ZhaW and ZheW conceived and designed the experiments. JY, HL, ZhaW, LY and QL conducted all the experiments. LY, QL, XN and TX contributed reagents, materials and analysis tools. $\mathrm{XN}$ and TX performed the experiments and were involved in the preliminary work. All authors read and approved the final manuscript.

\section{Ethics approval and consent to participate}

Not applicable.

\section{Patient consent for publication}

Not applicable.

\section{Competing interests}

The authors declare that they have no competing interests.

\section{References}

1. Li YJ, Li XL, Liang S, Fang LQ and Cao WC: Epidemiological features and risk factors associated with the spatial and temporal distribution of human brucellosis in China. BMC Infect Dis 13: 547, 2013.

2. Sanodze L, Bautista CT, Garuchava N, Chubinidze S, Tsertsvadze E, Broladze M, Chitadze N, Sidamonidze K, Tsanava S, Akhvlediani T, et al: Expansion of brucellosis detection in the country of Georgia by screening household members of cases and neighboring community members. BMC Public Health 15: 459, 2015.

3. Tuon FF, Gondolfo RB and Cerchiari N: Human-to-human transmission of Brucella-a systematic review. Trop Med Int Health 22: 539-546, 2017.

4. Zheng R, Xie S, Lu X, Sun L, Zhou Y, Zhang Y and Wang K: A systematic review and meta-analysis of epidemiology and clinical manifestations of human brucellosis in China. Biomed Res Int 2018: 5712920, 2018.

5. Elfaki MG, Alaidan AA and Al-Hokail AA: Host response to Brucella infection: Review and future perspective. J Infect Dev Ctries 9: 697-701, 2015.

6. Ma QL, Liu AC, Ma XJ, Wang YB, Hou YT and Wang ZH: Brucella outer membrane protein Omp25 induces microglial cells in vitro to secrete inflammatory cytokines and inhibit apoptosis. Int J Clin Exp Med 8: 17530-17535, 2015.

7. Zhang XG, Li GX, Zhao SS, Xu FL, Wang YH and Wang W: A review of dihydroartemisinin as another gift from traditional Chinese medicine not only for malaria control but also for schistosomiasis control. Parasitol Res 113: 1769-1773, 2014.
8. Dong F, Zhou X, Li C, Yan S, Deng X, Cao Z, Li L, Tang B, Allen TD and Liu J: Dihydroartemisinin targets VEGFR2 via the NF- $\mathrm{KB}$ pathway in endothelial cells to inhibit angiogenesis. Cancer Biol Ther 15: 1479-1488, 2014.

9. Zhang J, Guo L, Zhou X, Dong F, Li L, Cheng Z, Xu Y, Liang J, Xie Q and Liu J: Dihydroartemisinin induces endothelial cell anoikis through the activation of the JNK signaling pathway. Oncol Lett 12: 1896-1900, 2016

10. Im E, Yeo C, Lee HJ and Lee EO: Dihydroartemisinin induced caspase-dependent apoptosis through inhibiting the specificity protein 1 pathway in hepatocellular carcinoma SK-Hep-1 cells. Life Sci 192: 286-292, 2018.

11. Wang X, Lin P, Li Y, Xiang C, Yin Y, Chen Z, Du Y, Zhou D, Jin Y and Wang A: Brucella suis vaccine strain 2 induces endoplasmic reticulum stress that affects intracellular replication in goat trophoblast cells in vitro. Front Cell Infect Microbiol 6: 19, 2016.

12. Li M, Yu X, Guo H, Sun L, Wang A, Liu Q, Wang X and Li J: Bufalin exerts antitumor effects by inducing cell cycle arrest and triggering apoptosis in pancreatic cancer cells. Tumour Biol 35: 2461-2471, 2014.

13. Hang R, Zhang M, Ma S and Chu PK: Biological response of endothelial cells to diamond-like carbon-coated NiTi alloy. J Biomed Mater Res A 100: 496-506, 2012.

14. Zahedifard M, Faraj FL, Paydar M, Yeng Looi C, Hajrezaei M, Hasanpourghadi M, Kamalidehghan B, Abdul Majid N, Mohd Ali $\mathrm{H}$ and Ameen Abdulla M: Synthesis, characterization and apoptotic activity of quinazolinone Schiff base derivatives toward MCF-7 cells via intrinsic and extrinsic apoptosis pathways. Sci Rep 5: 11544, 2015.

15. Lin Z, Xu X, Zhao S, Yang X, Guo J, Zhang Q, Jing C, Chen S and He Y: Total synthesis and antimicrobial evaluation of natural albomycins against clinical pathogens. Nat Commun 9: 3445, 2018.

16. Pujol M, Castillo F, Alvarez C, Rojas C, Borie C, Ferreira A and Vernal R: Variability in the response of canine and human dendritic cells stimulated with Brucella canis. Vet Res 48: 72, 2017.

17. Miller CN, Smith EP, Cundiff JA, Knodler LA, Bailey Blackburn J, Lupashin V and Celli J: A brucella type IV effector targets the COG tethering complex to remodel host secretory traffic and promote intracellular replication. Cell Host Microbe 22: 317.e7-329.e7, 2017.

18. Fan L, Wu X, Jin C, Li F, Xiong S and Dong Y: MptpB promotes mycobacteria survival by inhibiting the expression of inflammatory mediators and cell apoptosis in macrophages. Front Cell Infect Microbiol 8: 171, 2018.

19. Kopeina GS, Prokhorova EA, Lavrik IN and Zhivotovsky B: Alterations in the nucleocytoplasmic transport in apoptosis: Caspases lead the way. Cell Prolif 51: e12467, 2018.

20. Julien O, Zhuang M, Wiita AP, O'Donoghue AJ, Knudsen GM, Craik CS and Wells JA: Quantitative MS-based enzymology of caspases reveals distinct protein substrate specificities, hierarchies, and cellular roles. Proc Natl Acad Sci USA 113: E2001-E2010, 2016.

21. Cui G, Wei P, Zhao Y, Guan Z, Yang L, Sun W, Wang S and Peng Q: Brucella infection inhibits macrophages apoptosis via Nedd4-dependent degradation of calpain2. Vet Microbiol 174: 195-205, 2014.

22. de Figueiredo P, Ficht TA, Rice-Ficht A, Rossetti CA and Adams LG: Pathogenesis and immunobiology of brucellosis: Review of Brucella-host interactions. Am J Pathol 185: 1505-1517, 2015.

23. Efferth T, Romero MR, Wolf DG, Stamminger T, Marin JJ and Marschall M: The antiviral activities of artemisinin and artesunate. Clin Infect Dis 47: 804-811, 2008.

24. Lam NS, Long X, Su XZ and Lu F: Artemisinin and its derivatives in treating helminthic infections beyond schistosomiasis. Pharmacol Res 133: 77-100, 2018.

25. Wang J, Zhou H, Zheng J, Cheng J, Liu W, Ding G, Wang L, Luo P, Lu Y, Cao H, et al: The antimalarial artemisinin synergizes with antibiotics to protect against lethal live Escherichia coli challenge by decreasing proinflammatory cytokine release. Antimicrob Agents Chemother 50: 2420-2427, 2016.

26. Jiang W, Li B, Zheng X, Liu X, Cen Y, Li J, Pan X, Cao H, Zheng J and Zhou H: Artesunate in combination with oxacillin protect sepsis model mice challenged with lethal live methicillin-resistant Staphylococcus aureus (MRSA) via its inhibition on proinflammatory cytokines release and enhancement on antibacterial activity of oxacillin. Int Immunopharmacol 11: 1065-1073, 2011.

This work is licensed under a Creative Commons Attribution-NonCommercial-NoDerivatives 4.0 International (CC BY-NC-ND 4.0) License. 